\title{
Isochrone Probability Functions for Old Stellar Systems
}

\author{
Peter A. Bergbusch \\ Department of Physics, University of Regina, \\ Regina, Saskatchewan, Canada, S4S 0A2
}

\begin{abstract}
The isochrone probability function (IPF) is derived from the slope of the mass-distance relation on an isochrone in $\mathcal{M}_{\odot} / \mathrm{mag}$, where the distance along the isochrone is computed with respect to some arbitrary, well-defined point. IPFs contain the information needed to calculate both luminosity functions and color functions, and they provide a straightforward way of generating synthetic stellar populations.
\end{abstract}

\section{Mathematical Formalism}

The mathematical formalism for the derivation of accurate IPFs parallels that for the derivation of luminosity functions presented in Bergbusch \& VandenBerg (1992) (hereafter BV92), except that the distance along an isochrone is used as the observable coordinate instead of luminosity.

In the $\log L-\log T_{\text {eff }}$ plane, the distance along an isochrone is defined by

$$
d \mathcal{D}=\left[c_{1}(d \log L)^{2}+c_{2}\left(d \log T_{\mathrm{eff}}\right)^{2}\right]^{1 / 2}
$$

where $c_{1}$ and $c_{2}$ are chosen to stretch the subgiant branch (SGB) and to obtain a convenient range of distances. The slope of the mass-distance relation is then obtained from

$$
\frac{d \mathcal{D}}{d \mathcal{M}}=\left[c_{1}\left(\frac{\partial \log L}{\partial \mathcal{M}}\right)^{2}+c_{2}\left(\frac{\partial \log T_{\text {eff }}}{\partial \mathcal{M}}\right)^{2}\right]^{1 / 2}
$$

Consequently, equation (6) from BV92 is recast as

$$
\phi(\mathcal{D})=N(\mathcal{M})\left(\left.\frac{d \mathcal{D}}{d \mathcal{M}}\right|_{t}\right)^{-1},
$$

where $\phi(\mathcal{D})$ is the isochrone probability function, and the evaluation of the partial derivatives in equation (2) is performed as described in BV92.

In the observer's plane, distance is calculated with respect to a point on the SGB $0.05 \mathrm{mag}$ redward of the main sequence turnoff (regardless of color index). A convenient definition of distance is

$$
d D=\left[d M_{i}^{2}+16 d C_{i k}^{2}\right]^{1 / 2},
$$




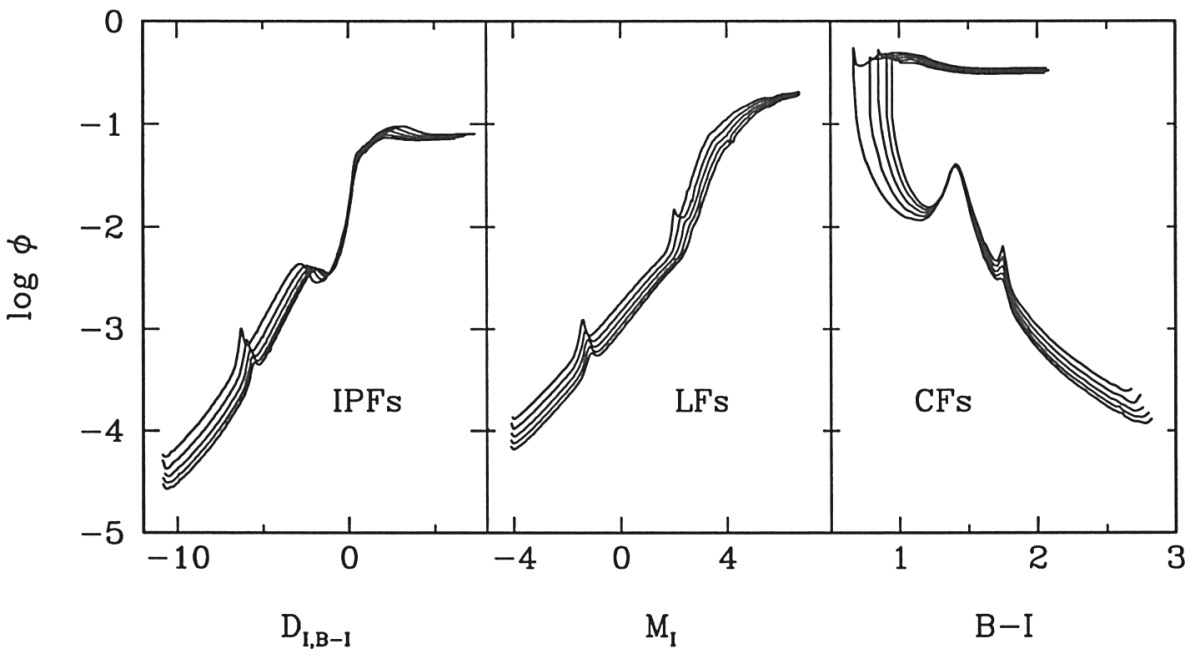

Figure 1. IPFs, LFs, and CFs for the ages 8-16(2) $\mathrm{Gyr}$ and $[\mathrm{Fe} / \mathrm{H}]=$ -2.14 , plotted for the $I$ passband and the $B-I$ color index.

where $M_{i}$ is the absolute magnitude in the $i^{\text {th }}$ passband and $C_{i k}$ is the color index for passbands $i$ and $k$. A $\mathcal{D}-D$ calibration is used to translate bin limits from the observer's plane to the theoretical plane, and integrations across bins are performed on the theoretical plane.

\section{Results}

The Akima spline (Akima 1970) has been utilized throughout the interpolation relations to obtain the isochrone variables (mass, luminosity, effective temperature) and their derivatives. The leftmost panel of Fig. 1 shows the differential IPFs on the observer's plane for $[\mathrm{Fe} / \mathrm{H}]=-2.14$, binned at 0.05 mag intervals, transformed to the observer's plane. The luminosity functions (LFs) plotted in the middle panel (in 0.05 mag bins) and the color functions (CFs) plotted in the rightmost panel (in $0.02 \mathrm{mag}$ bins) were both derived directly from the IPF. The small wiggles which appear throughout the IPFs, LFs, and CFs are mostly due to inadequacies in the bolometric corrections and in the color $-T_{\text {eff }}$ relations. As pointed out by Bergbusch \& VandenBerg (1997), the CF of the subgiant region can be used to provide a distance-independent constraint on cluster ages.

\section{References}

Akima, H. 1970, JACM, 17, 589

Bergbusch, P., \& VandenBerg, D. A. 1992, ApJS, 81, 163

Bergbusch, P., \& VandenBerg, D. A. 1997, AJ, 114, 2604 